\title{
Inter- and intra-individual variability in food intake of elderly people in Perugia (Italy)
}

\author{
BY R. BORRELLI, ${ }^{1,2}$ M. S. SIMONETTI ${ }^{1}$ AND F. FIDANZA ${ }^{1}$ \\ ${ }^{1}$ Institute of Nutrition, University of Perugia, Perugia, Italy, \\ ${ }^{2}$ National Cancer Institute 'Fondazione G. Pascale', Naples, Italy'
}

(Received 25 February 1991-Accepted 30 July 1991)

\begin{abstract}
It has been shown that each individual has a considerable day-to-day variation (intra-individual variation) in his or her level of food consumption. A large intra-individual variation has adverse effects on the reliability of research studies. The effect of the intra-individual variation can be minimized by taking food intake records over several days. An increase in the number of days entails higher costs, and this could limit the practicability of the study. In the recent literature on the methodology of dietary surveys, there is a growing interest in the estimation of the number of days required to conduct a reliable dietary survey. Recent developments in statistical theory allow the problem of large intra-individual variability to be overcome. These new statistical techniques require knowledge of the intra- and interindividual variability and the appropriate adjustment of the statistical results. The aim of the present study was to evaluate the number of days of recorded intake required to obtain an estimate of the components of variance (the inter-individual and the intra-individual variability) focusing on foods rather than nutrients.
\end{abstract}

Components of variance: Dietary surveys: Elderly

In the recent literature on the methodology of dietary surveys, there has been a growing interest in the estimation of the number of days required to assess dietary status of populations, or to study the relationship between diet and disease with good precision (Liu et al. 1978; Black et al. 1983; Marr \& Heady, 1986; Basiotis et al. 1987; Nelson et al. 1989).

The basic difficulty in obtaining a precise estimate of habitual food intake by the diet record method is the tendency of each individual to vary considerably from day to day his level and pattern of food consumption (intra-individual variation; Bingham, 1987). This variation can be greater than the inter-individual variation (the variation between different individuals).

Intra-individual variation has adverse effects on the reliability of research studies. It reduces the probability of detecting a significant relationship between diet and disease (Liu et al. 1978); it leads to biased estimation of upper and lower centiles of food intake distribution (Beaton, 1988); it leads to misclassification when ranking individuals on the basis of their intakes, etc. (Marr \& Heady, 1986).

Two solutions have been proposed to overcome the problem of large daily variation which occurs in the diet record method (National Research Council, 1986; Rosner \& Willett, 1988; Borrelli et al. 1989; Liu, 1989).

The first is to collect the recorded intake over a large number of days, since averaging several days' observations reduces the intra-individual variation. Bingham (1987) observed that up to $45 \mathrm{~d}$ recorded intake are required to obtain reliable estimates of intake for some particular nutrients (such as cholesterol). However, she stated that in general at least $14 \mathrm{~d}$ of recorded intake are required for most of the nutrients, depending on the aim of the study. 
An alternative solution is to compensate the statistical results for the intra-individual variation, but this method requires knowledge of the intra-individual and inter-individual variation. By using this alternative statistical solution, it is not necessary to take so many replicates of recorded intake, but only a few replicates to obtain an estimate of the interindividual and intra-individual variation.

The aim of the present study was to evaluate the number of days of recorded intake required to obtain a reliable estimate of the components of variance (the inter-individual and the intra-individual variation).

FXPERIMENTAL PROCEDURES

Design of study

Twenty-four (ten men and fourteen women) elderly subjects were recruited from a home for the elderly for the present study. Details of this sample have been reported previously (Fidanza et al. 1988).

These elderly subjects were able to choose their menus every day (for the following day) which enabled them to preserve their dietary habits both in terms of food choice and portion size. Food intake was estimated by the weighing method for three non-consecutive weeks. Only food items and not nutrients were considered in the present study in order to exclude the source of variation due to the error in the food composition tables (National Research Council, 1986). Furthermore, the analysis of food allows a better understanding of where the variation comes from.

\section{Statistical procedures}

For each food examined, three statistical parameters were estimated: the variation between individuals, the intra-individual variation and the mean of intakes. The inter- and the intra-individual variations were estimated by means of components of variance analysis (Cochran, 1968; Armitage \& Berry, 1987). For each food item, the components of variance were estimated twenty times on food intake measures on the basis of the average of the first $2 \mathrm{~d}$ (the first time), and then adding the following days of collection, $1 \mathrm{~d}$ at a time until the 21 st day of collection.

Food intake is shown for each of the three main meals of the day (breakfast, lunch and dinner) that these subjects consumed.

\section{RESULTS}

In Fig. 1 the consumption of milk and meat (canned, fresh, sausages, etc.) during breakfast is shown. The estimation of the mean and the inter- and intra-individual variation for milk became stable after a few days of recorded intake. The same findings were found for other foods such as cereals (data not shown). The estimation of mean, inter- and intra-individual variation was more difficult for meat (canned, sausages, etc.) consumed at breakfast. In fact, there was no consumption of these food items at all until the ninth day.

Fig. 2(a and b) shows foods consumed at lunch. The estimation of the mean and the inter- and intra-individual variation became stable after a few days of recorded intakes for cereals, vegetables (Fig. 2(a)), cheeses, fruits, potatoes, eggs, and meat (not shown). The estimation of mean, inter- and intra-individual variation of fish and milk intake (Fig. 2(b)) seemed to vary with additional days of recorded intake.

Fig. 3( $\mathrm{a}$ and $\mathrm{b}$ ) shows the food consumed at dinner. Fig. 3(a) shows the estimation of the mean and the inter-and intra-individual variation of cheese and vegetable intake. The estimation of these statistical parameters became stable after a few days of recorded intake 

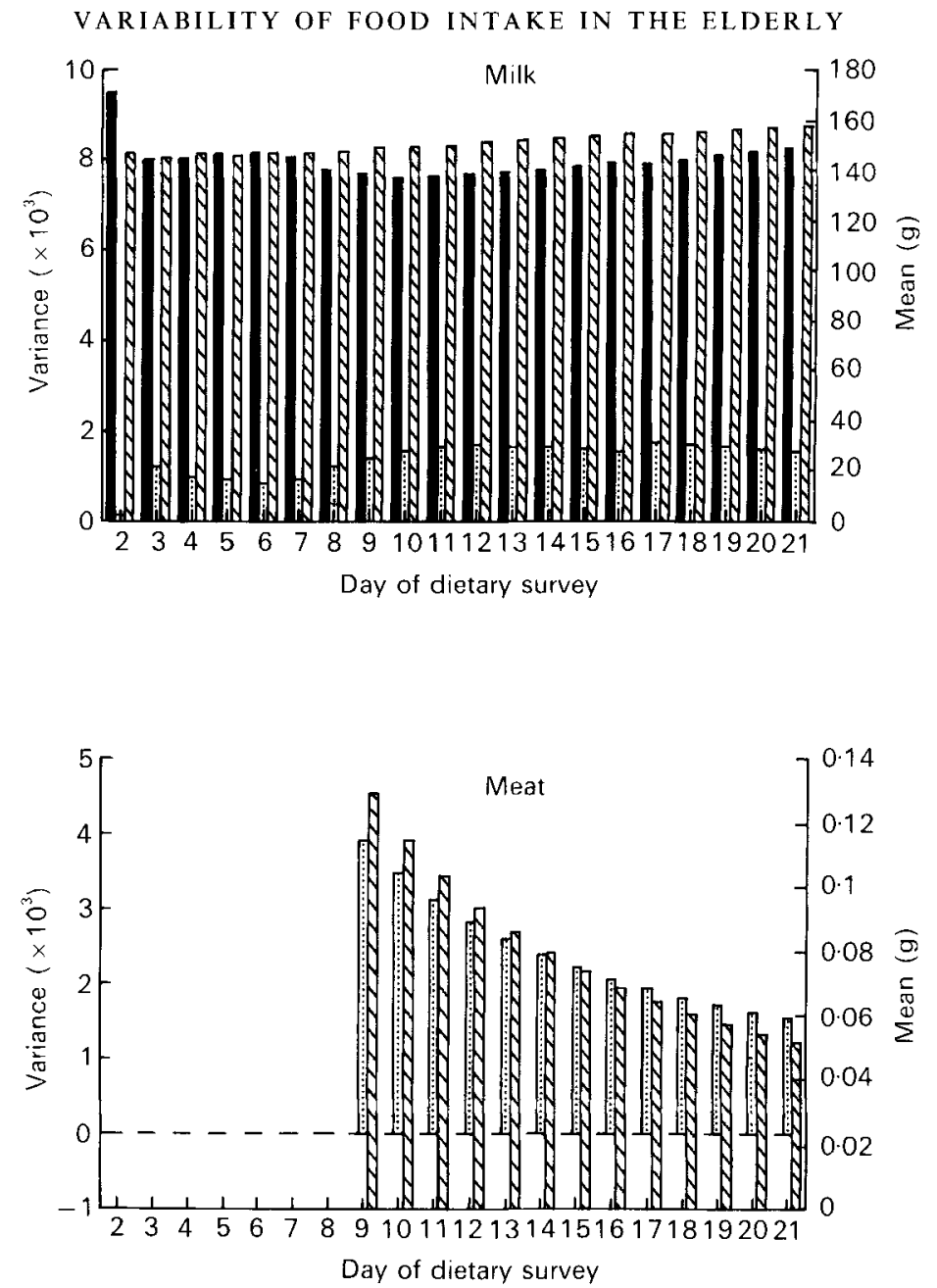

Fig. 1. Mean $(\nabla)$, inter- $(\boldsymbol{D})$ and intra $(\square)$-individual variation for milk and meat consumption of elderly Italian subjects at breakfast estimated on food intake measurements on the basis of the average of the first $2 \mathrm{~d}$ (the first time), and then adding the following days of collection, $1 \mathrm{~d}$ at a time until the 21 st day of collection. For details of subjects and procedures, see p. 4.

(usually less than $7 \mathrm{~d}$ ). The same findings were recorded for meat, milk, cereals, fruits and potatoes (data not shown). Fig. 3(b) shows that the estimation of these parameters was more difficult for legume and meat consumption.

\section{DISCUSSION}

A question that is frequently asked is "how many days of food recorded intake are required to achieve a sufficient precision for the purpose of dietary surveys?'.

The increase in the number of days entails higher costs, which could limit the practicability of the study. Several authors have tried to determine the number of days required to achieve good precision (Liu et al. 1978; Black et al. 1983; Marr \& Heady, 1986; Basiotis et al. 1987; Nelson et al. 1989). They found that more than $7 \mathrm{~d}$ of recorded intake are usually required for most nutrients, depending on the aim of the study, the nutrient and so on (Bingham, 1987). 
(a)
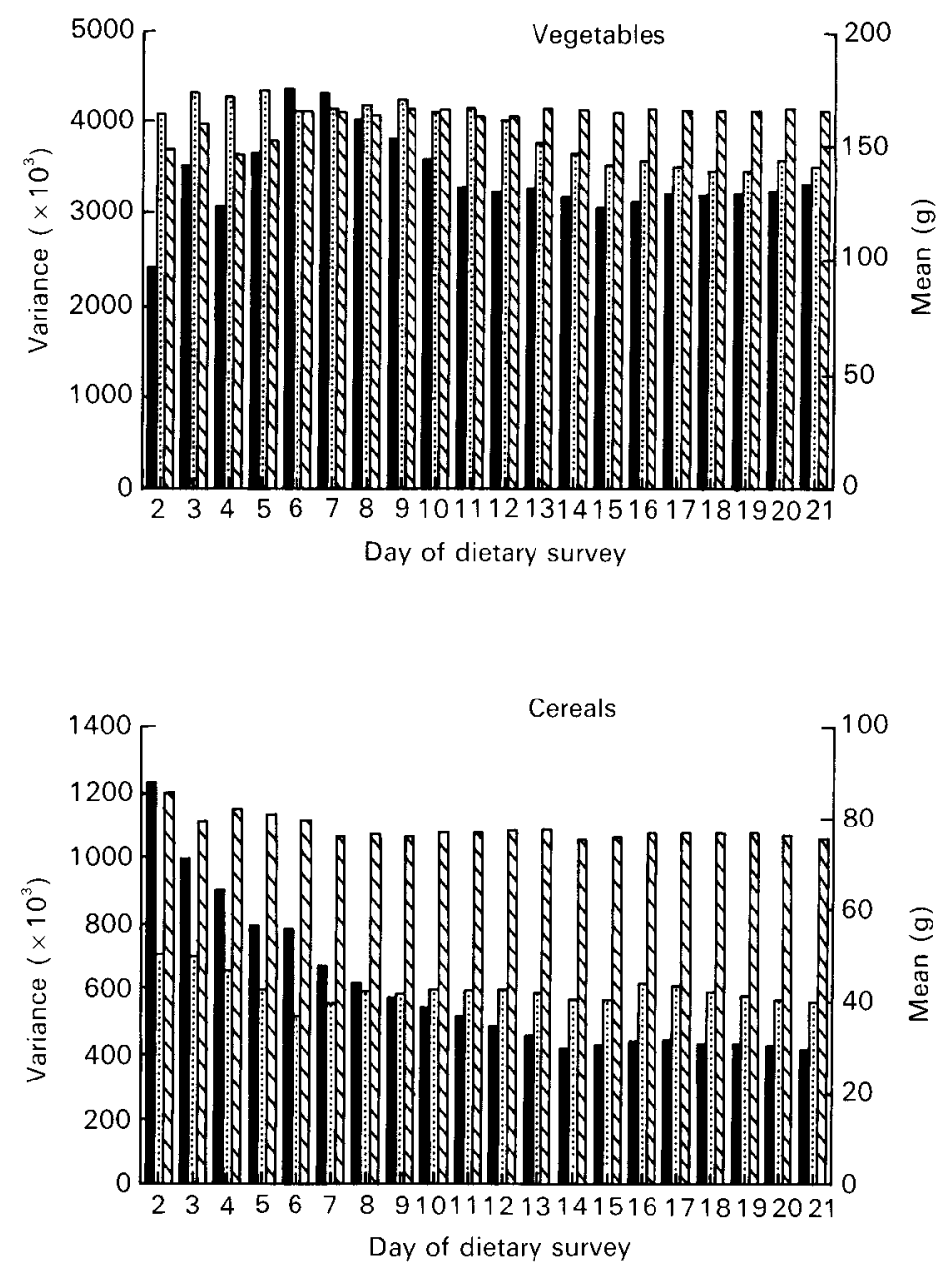

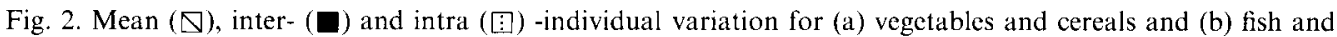
milk consumption of elderly Italian subjects at lunch estimated on food intake measurements on the basis of the average of the first $2 \mathrm{~d}$ (the first time), and then adding the following days of collection, $1 \mathrm{~d}$ at a time until the $2 \mathrm{lst}$ day of collection. For details of subjects and procedures, see p. 4.

Recent developments in statistical theory allow the problem of large intra-individual variation to be overcome (National Research Council, 1986; Rosner \& Willett, 1988; Borrelli et al. 1989; Liu, 1989). These new statistical techniques require knowledge of the intra- and inter-individual variation and adjust statistical results for them. Methods are available for simple correlation, simple linear regression, logistic regression and distributional analysis (Liu, 1988; Rosner \& Willett, 1988; Rosner et al. 1990). These statistical techniques assume that the intra-individual variation is independent of the level of intake (i.e. an individual is assumed to vary randomly in his or her own true daily mean values), that the distribution of intake is 'normal' and so on (Borrelli et al. 1989).

By using these new statistical techniques, the limiting factor in the dietary surveys design becomes a reliable estimation of the variance components.

This issue has usually been concerned with assessing variations in the consumption of 

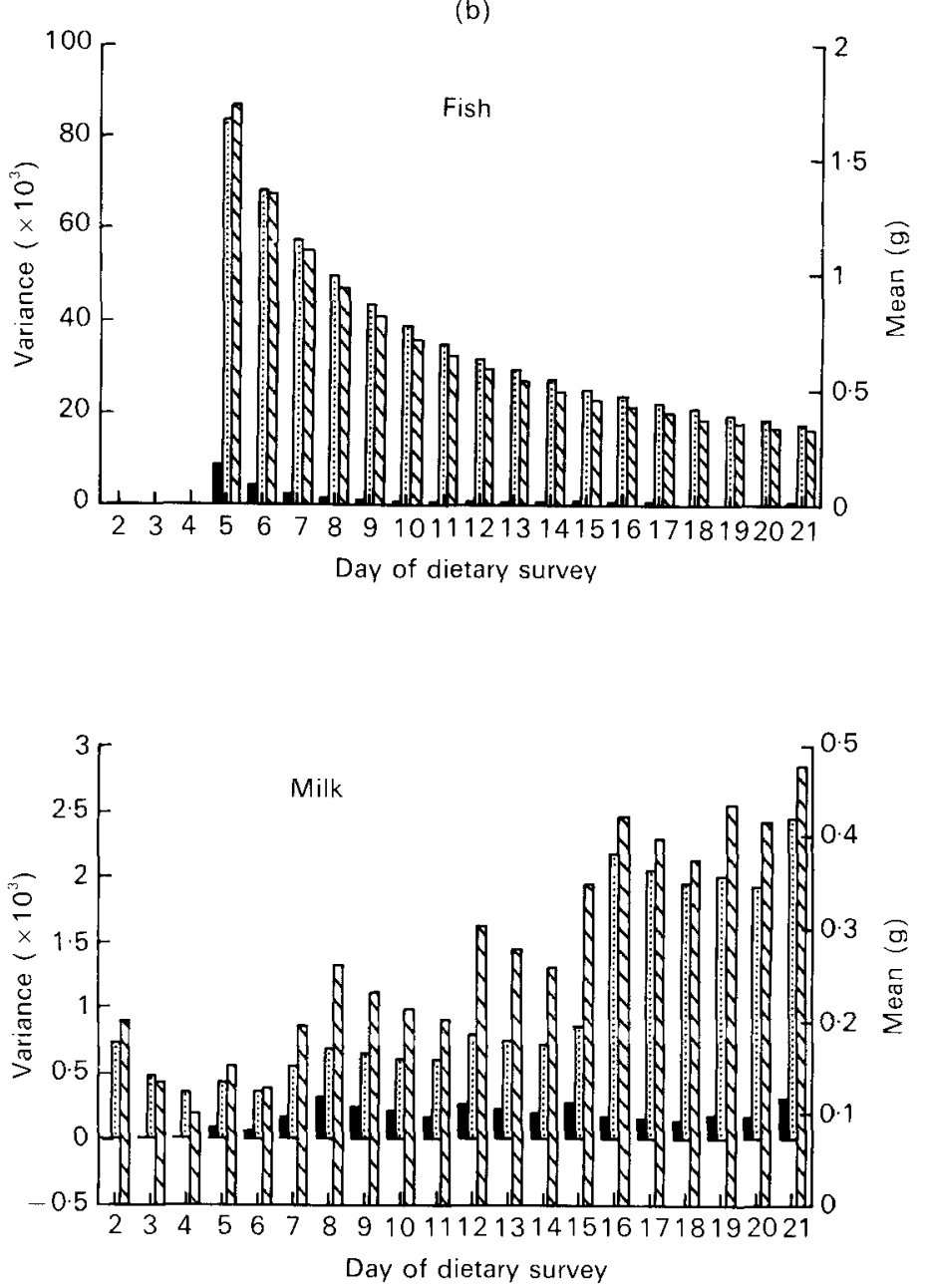

Fig. 2. For legend see opposite.

nutrients. In the present paper the variations in the consumption of foods are considered to permit a better understanding of where these variations come from.

In the present paper the intra-individual and inter-individual variations in food consumption are estimated by using from 2 to $21 \mathrm{~d}$ of recorded intake data from a sample of elderly people from Perugia. The data are subdivided according to the three main meals of the day. The estimation of intra-individual and inter-individual variation seems to be consistent and to fall well within reasonable ranges for most of the foods when evaluated by measurements based on the average of a few days of recorded intake or many days (Figs. 2(a) and 3(a)). For these foods, the addition of more recorded days of food intake will not improve the adequacy of the estimation of the three fundamental statistical parameters. Food intake data recorded on some foods, certain less commonly eaten foods (Figs. 2(b) and $3(b))$, at lunch or dinner seem to give some problems. In fact, the estimation of the mean and the inter-individual and the intra-individual variation do vary if more days of data 
(a)
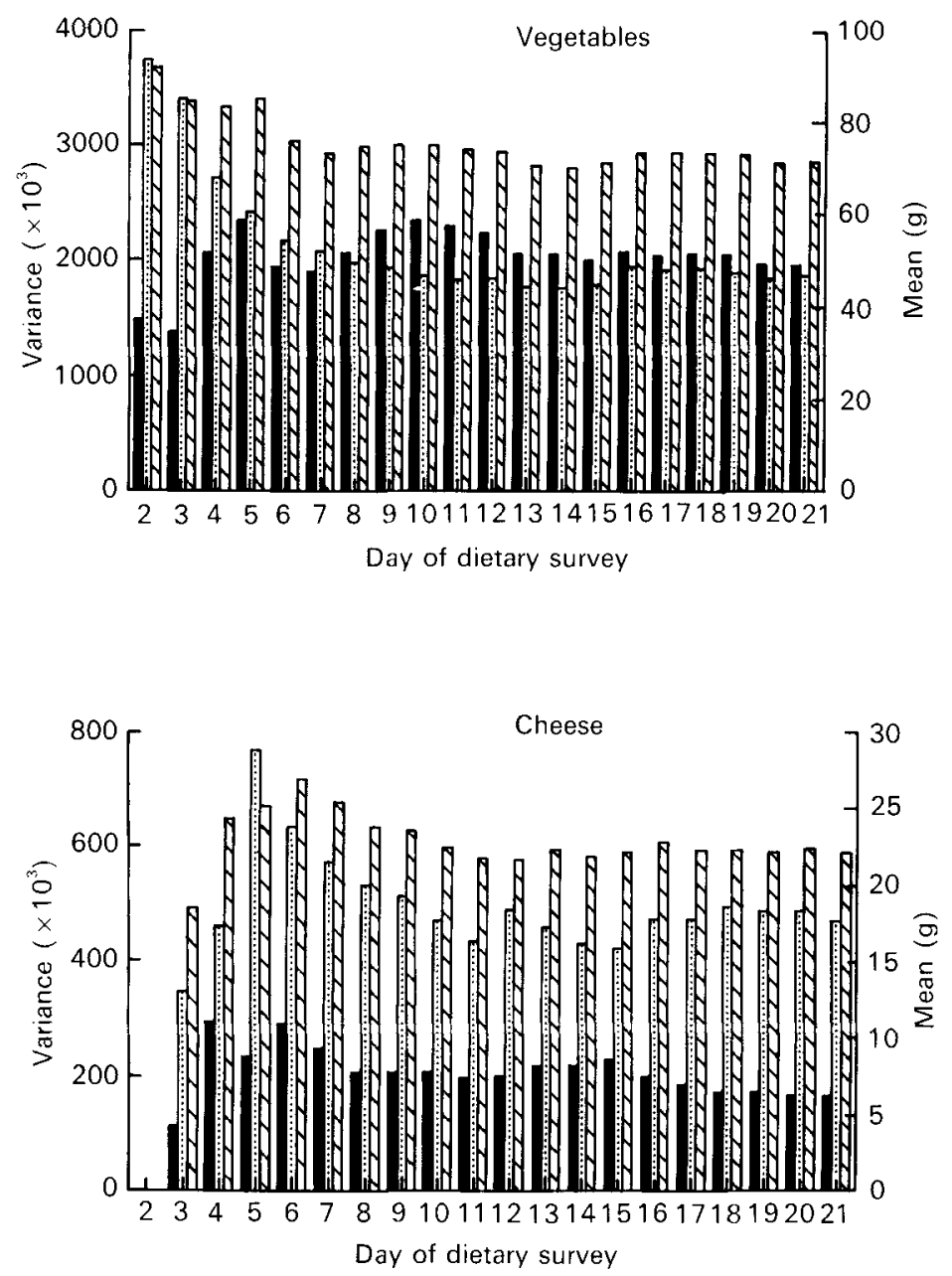

Fig. 3. Mean $(\nabla)$, inter- $(\boldsymbol{D})$ and intra (:) -individual variation for (a) vegetables and cheese and (b) meat and legume consumption of elderly Italian subjects at dinner estimated on food intake measurements on the basis of the average of the first $2 \mathrm{~d}$ (the first time), and then adding the following days of collection, $\mathrm{I} d$ at a time until the 21 st day of collection. For details of subjects and procedures, see p. 4.

collection are added. Also, the intake of meat (canned, fresh, sausages, etc.) at breakfast gives some problems for the estimation of variance components.

In these cases, the fluctuation in the estimation of the variance components between the measurements obtained for just a few days of recorded intake and those obtained by adding more days does not seem to be very large. It may well be that this fluctuation is not a problem in terms of nutrients because the uncommonly eaten foods contribute very little to the total daily intake. However, the impact of these discrepancies should be studied.

\section{Conclusions}

A few days of food intake record are sufficient to obtain an estimate of the components of variance and of the mean of an elderly population in Perugia for most of the food items. The estimates can be effectively used in statistical analyses to obtain a reliable study. 
(b)
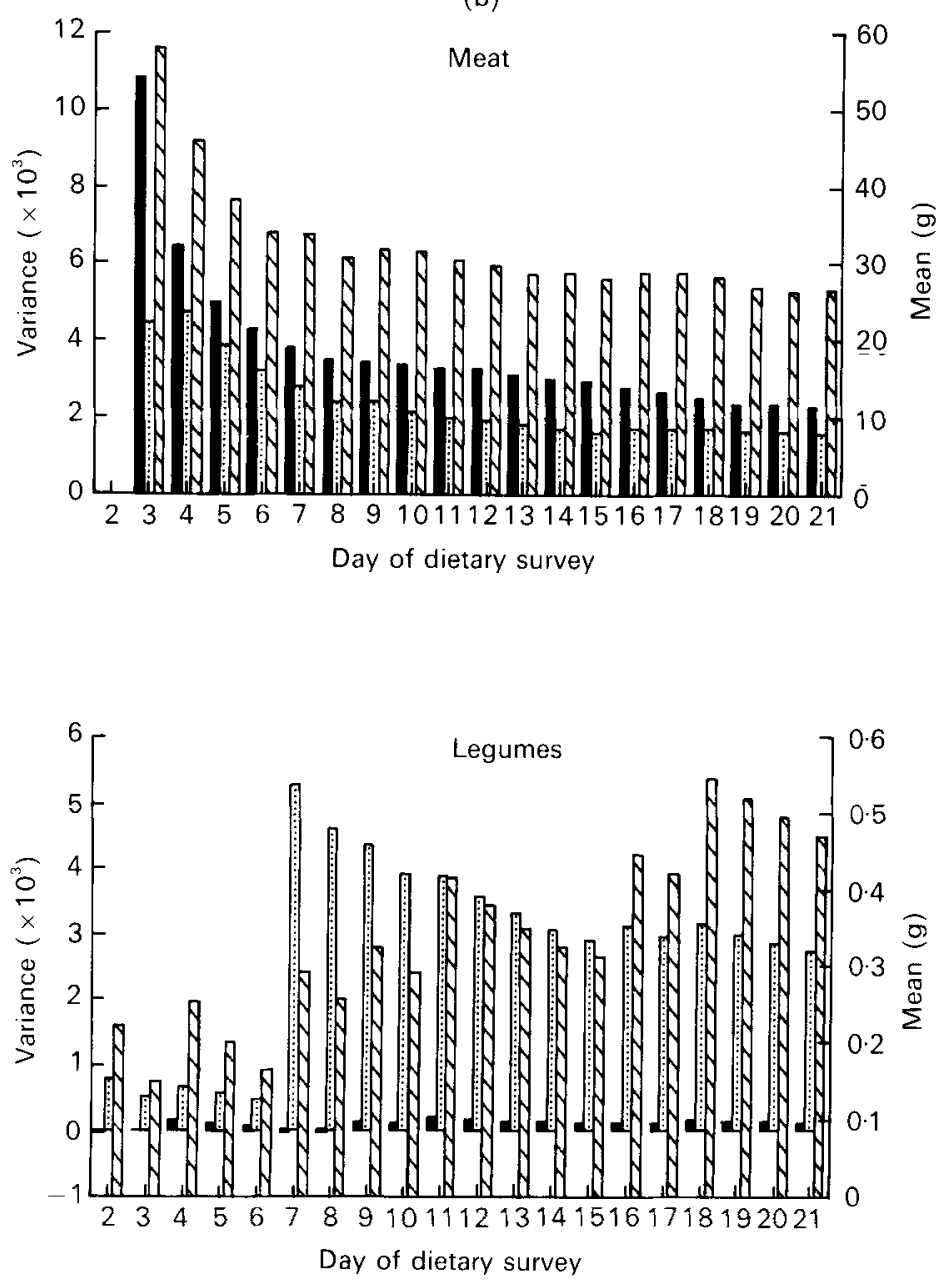

Fig. 3. For legend see opposite.

\section{REFERENCES}

Armitage, P. \& Berry, G. (1987). Statistical Methods in Medical Research, 2nd ed. Oxford: Blackwell Scientific Publications.

Basiotis, P. P., Welsh, S. O., Cronin, F. J., Kelsay, J. L. \& Mertz, W. (1987). Number of days of food intake records required to estimate individual and group nutrient intake by men and women as determined from one year's daily dictary records. Journal of Nutrition 117, 1638-1641.

Beaton, G. H. (1988). Nutrient intake and population data. Proceedings of the Nutrition Society 47, 6378.

Bingham, S. A. (1987). The dietary assessment of individuals; methods, accuracy, new techniques and recommendations. Nutrition Abstracts and Reviews 57, 705-742.

Black, A. E., Cole, T. J., Wiles, S. J. \& Winter, F. (1983). Daily variation in food intake in infants from 2 to 18 months. Human Nutrition: Applied Nutrition 37A, 448-458.

Borrelli, R., Cole. T. J., Di Biase, G. \& Contaldo, F. (1989). Some statistical considerations on dietary assessment methods. European Journal of Clinical Nutrition 43, 453-463.

Cochran, W. G. (1968). Errors of measurement in statistics. Technometrics 10, 637 666.

Fidanza, F., Coli, R., Damiani, P., Fiorini, D., Maurizi, A. \& Simonetti, M. S. (1988). Nutritional status of a group of institutionalized elderly people in Perugia (Italy). International Journal of Vitamin and Nutrition Research 58, 85-90. 
Liu, K. (1988). Measurement error and its impact on partial correlation coefficient and multiple linear regression analyses. American Journal of Epidemiology 12, 864-874.

Liu, K. (1989). Consideration of and compensation for intraindividual variability in nutrient intake. In Epidemiology, Nutrition and Health, pp. 87-97 [L. Kohlmeier and H. Helsing, editors]. London: Smith-Gordon.

Liu, K., Stamler, J., Dyer, A., McKeever, J. \& McKeever, P. (1978). Statistical methods to assess and minimize the role of intraindividual variability in obscuring the relationship between dietary lipid and serum cholesterol. Journal of Chronic Diseases 31, 399-418.

Marr, J. W. \& Heady, J. A. (1986). Within- and between-person variation in dietary surveys: number of days needed to classify individuals. Human Nutrition: Applied Nutrition 40A, 347-364.

National Research Council (1986). Nutrient Adequacy: Assessment Using Food Consumption Surveys. Report of the Food and Nutrition Board. Washington, DC: National Academy of Sciences.

Nelson, M., Black, A. E., Morris, J. A. \& Cole, T. I. (1989). Between- and within-person variation in nutrient intake from infancy to old age: estimating the number of days required to rank dietary intakes with desired precision. American Journal of Clinical Nutrition 50, 155--167.

Rosner, B., Spiegelman, D. \& Willett, W. C. (1990). Correction of logistic regression relative risk estimates and confidence intervals for measurement error: the case of multiple covariates measured with error. American Journal of Epidemiology 132, 734-745.

Rosner, B. \& Willett, W. C. (1988). Interval estimates for correlation coefficient corrected for within-person variation: implications for study design and hypothesis testing. American Journal of Epidemiology 127, 377-386. 